\title{
Guaranteed Cost Control Research for Networked Control System with Time-Varying Sampling Period
}

\author{
Nana Wang, Nan Xie* \\ College of Computer Science and Technology, Shandong University of Technology, Zibo, China \\ Email address: \\ 1748462348@qq.com (Nana Wang),xienan@sdut.edu.cn (Nan Xie) \\ ${ }^{*}$ Corresponding author
}

To cite this article:

Nana Wang, Nan Xie. Guaranteed Cost Control Research for Networked Control System with Time-Varying Sampling Period. International Journal of Sensors and Sensor Networks. Vol. 9, No. 1, 2021, pp. 38-44. doi: 10.11648/j.ijssn.20210901.16

Received: April 28, 2021; Accepted: May 21, 2021; Published: May 27, 2021

\begin{abstract}
In practical industrial applications, the networked control system is often affected by external factors and internal components in operation, which leads to the fluctuation of some data transmitted by the system and reduces the system performance. Therefore, to ensure the normal operation of the system is the key, on this basis, we also need to make the system satisfy certain performance indicators. In this paper, guaranteed cost control for networked control systems with time delay and time-varying sampling period is studied. By constructing a more general system model, we give a design method of guaranteed performance controller to make the closed-loop system asymptotically stable within the allowable range and and satisfies certain performance indicators. Firstly, we construct the reasonable Lyapunov function and performance index function. According to the relevant lemma, the sufficient conditions for the stability of the system are obtained by equivalent transformation of LMI. Then, we design a guaranteed performance controller to minimize the upper bound of the system performance index. In the design process, more free variables are introduced, and the convexity of the function is used for equivalent transformation as far as possible to make the results less conservative. Finally, we use MATLAB to simulate and prove that the method is feasible.
\end{abstract}

Keywords: Networked Control System, Time-Varying Sampling Period, LMI, Guaranteed Cost Control

\section{Introduction}

The emergence of networked control system brings great convenience to the control field. The network is introduced into the traditional control system to create a more automatic and intelligent control mode $[1,2]$. However, due to the influence of various uncertain factors, some parameters of the controller will fluctuate, which will affect the normal operation of the system. The parameters of the controller should be analyzed when designing the controller. Therefore, the concept of guaranteed performance control came into being [3]. Generally speaking, guaranteed cost control refers to the ability to master the optimal method of the system to achieve the expected goal, and make the performance index not exceed the specified range [4]. Its essence is to design acontroller for a system with parameter uncertainty and certainconstraint conditions, by using LMI method, we carry out equivalent transformation and get the stable condition, so that the system can not only run stably, but also meet the requirement that the system performance index is less than the upper bound value of the performance index provided. Therefore, how to design a guaranteed performance controller with better performance is a research topic that attracts much attention.

In addition, many achievements have been made in the study of time-varying sampling period [5-7]. Constant sampling periods were investigated in early control studies, where the sampling period was set to a constant, which was also the easiest method $[8,9]$. However, sample period jitter is inevitable due to computer hardware facilities and network load, so it is clearly unreasonable to set the sample period to a constant at all times. Therefore, in order to make the designed system more practical, it is necessary to study the time-varying sampling period. Literature [10-12] designed a memoryless state feedback controller to ensure the performance of the system. Literature [13] considered the time-delay, and the state feedback controller was designed by using time-varying observer, LMI and Lyapunov function. The practicability of this method was demonstrated by experiments. On this basis, literature [14] gave a design 
method for a system $\mathrm{H} \infty$ controller, which modeled NCSs with variable sampling periods as switching systems, designed a reasonable $\mathrm{H} \infty$ controller, and optimized it using LMIs constraints. For nonlinear time-varying delay systems, Literature [15] studied the fuzzy sampling optimal control problem by using input delay and free weight matrix method, and then gave the conditions for the existence of optimal controllers. In order to make the bandwidth utilization higher, the active variable sampling method was used to switch the active variable sampling period within a certain period, and the controller of NCSs with time-delay was designed in the case of packet loss [16].

In this paper, guaranteed cost control for NCSs with time delay and variable sampling period is considered. In Section 2, the corresponding uncertain systems are described, and a more general system model is given, which is transformed into parameter uncertainty to establish a more general system model. In Section 3, we analyze the stability of the proposed model and give the design method of guaranteed cost controller. In Section 4, we use MATLAB to simulation, and give specific numerical examples to prove the effectiveness of the numerical proof method. Section 5 is the summary of the full text.

\section{Problem Statement and Modeling}

It is assumed that the state model of the controlled object is:

$$
\left\{\begin{array}{l}
\dot{x}(t)=A_{k} x(t)+B_{k} u(t) \\
y(t)=C x(t)
\end{array}\right.
$$

where $x(t)$ is the state of the system, $u(t)$ is the input of the controlled object, $y(t)$ is the output of the controlled object. $A_{k}, B_{k}$ and $C$ are well-dimensional matrices. For the convenience of description, select the time driven on the sensor side, and use the event driven on the controller and actuator side. And assumes that the time delay $h_{k}$ and the sampling period $t_{k}$ are bounded and time-varying uncertain, and satisfying $h_{k}=t_{k+1}-t_{k}, k \in N$.

In practical industrial application, due to the influence of various factors, the sampling period will inevitably shake, and there is often time-varying uncertain delay. In general, the total delay is $\tau_{k}=\tau_{s c}+\tau_{c a}+\tau_{c}$, where $\tau_{c}$ is the time delay generated when the controller calculates the control quantity, which can be ignored in general. Therefore, it can be assumed that the delay $\tau_{k}$ and the sampling period $h_{k}$ are bounded and time-varying uncertain, satisfying $\tau_{k} \leq h_{k}$, where $h_{k} \in\left[h_{\min }, h_{\max }\right], \tau_{k} \in\left[\tau_{\min }, \tau_{\max }\right]$.

According to the above analysis and hypothesis, the state equation of the controlled object is discretized, and the following discrete equation can be obtained:

$$
\begin{aligned}
& x_{k+1}=A_{0} x_{k}+H_{0}\left(h_{k}, \tau_{k}\right) u_{k}+H_{1}\left(h_{k}, \tau_{k}\right) u_{k-1} . \\
& y_{k}=C x_{k}
\end{aligned}
$$

where

$$
\begin{aligned}
& A_{0}=e^{A T}, \\
& H_{0}\left(h_{k}, \tau_{k}\right)=\int_{0}^{h_{k}-\tau_{k}} e^{A_{s}} B d s, \\
& H_{1}\left(h_{k}, \tau_{k}\right)=\int_{h_{k}-\tau_{k}}^{h_{k}} e^{A_{s}} B d s .
\end{aligned}
$$

For the sake of description, we define the augmented matrix vector $z(k)=\left[\begin{array}{ll}x^{T}(k) & u^{T}(k-1)\end{array}\right]^{T}$. Therefore, from (2) we have:

$$
z(k+1)=\left[\begin{array}{cc}
A_{0} & H_{1}\left(h_{k}, \tau_{k}\right) \\
0 & 0
\end{array}\right] z(k)+\left[\begin{array}{c}
H_{0}\left(h_{k}, \tau_{k}\right) \\
I
\end{array}\right] u(k)
$$

Assume that

$$
\begin{aligned}
& A\left(h_{k}, \tau_{k}\right)=\left[\begin{array}{cc}
A_{0} & H_{1}\left(h_{k}, \tau_{k}\right) \\
0 & 0
\end{array}\right]=\left[a_{i j}\right]_{(n+p) \times(n+p)} \\
& B\left(h_{k}, \tau_{k}\right)=\left[\begin{array}{c}
H_{0}\left(h_{k}, \tau_{k}\right) \\
I
\end{array}\right]=\left[b_{i j}\right]_{(n+p) \times p}
\end{aligned}
$$

where $a_{i j}$ and $b_{i j}$ are the corresponding elements of matrix $A\left(h_{k}, \tau_{k}\right)$ and $B\left(h_{k}, \tau_{k}\right)$ respectively. Since $h_{k}$ and $\tau_{k}$ are time-varying indeterminate and bounded, $A\left(h_{k}, \tau_{k}\right)$ and $B\left(h_{k}, \tau_{k}\right)$ are also time-varying indeterminate and bounded.

Assume that

$$
\begin{aligned}
& A^{m}=\left[a_{i j}^{m}\right] \in R^{(n+p) \times(n+p)}, A^{M}=\left[a_{i j}^{M}\right] \in R^{(n+p) \times(n+p)}, \\
& a_{i j}^{m} \leq a_{i j} \leq a_{i j}^{M}, \\
& B^{m}=\left[b_{i j}^{m}\right] \in R^{(n+p) \times p}, B^{M}=\left[b_{i j}^{M}\right] \in R^{(n+p) \times p}, \\
& b_{i j}^{m} \leq b_{i j} \leq b_{i j}^{M},
\end{aligned}
$$

where,

$$
\begin{aligned}
& a_{i j}^{m}=\min a_{i j}\left(h_{k}, \tau_{k}\right), a_{i j}^{M}=\max a_{i j}\left(h_{k}, \tau_{k}\right), \\
& b_{i j}^{m}=\min b_{i j}\left(h_{k}, \tau_{k}\right), b_{i j}^{M}=\max b_{i j}\left(h_{k}, \tau_{k}\right),
\end{aligned}
$$

Then, the system (4) can be written as

$$
z(k+1)=A\left(h_{k}, \tau_{k}\right) z(k)+B\left(h_{k}, \tau_{k}\right) u(k)
$$

where, $A^{m} \leq A \leq A^{M}, B^{m} \leq B \leq B^{M}$.

Assume that

$$
A_{0}=\left(A^{m}+A^{M}\right) / 2, \quad B_{0}=\left(B^{m}+B^{M}\right) / 2,
$$




$$
\begin{aligned}
D_{1}= & {\left[\sqrt{\lambda_{11}} e_{1}, \ldots, \sqrt{\lambda_{1(n+p)}} e_{1}, \sqrt{\lambda_{21}} e_{2}, \ldots, \sqrt{\lambda_{2(n+p)}} e_{2}, \ldots, \sqrt{\lambda_{(n+p) 1}} e_{(n+p)}, \ldots, \sqrt{\lambda_{(n+p)(n+p)}} e_{n+p}\right], } \\
F_{1}= & \operatorname{diag}\left(\varepsilon_{11}, \ldots, \varepsilon_{1(n+p)}, \varepsilon_{21}, \ldots, \varepsilon_{2(n+p)}, \varepsilon_{(n+p) 1}, \ldots, \varepsilon_{(n+p)(n+p)}\right),\left|\varepsilon_{i j}\right| \leq 1,1 \leq i, j \leq n+p, \\
E_{1}^{T}= & {\left[\sqrt{\lambda_{11}} e_{1}, \ldots, \sqrt{\lambda_{1(n+p)}} e_{(n+p)}, \sqrt{\lambda_{21}} e_{1}, \ldots, \sqrt{\lambda_{2(n+p)}} e_{(n+p)}, \ldots, \sqrt{\lambda_{(n+p) 1}} e_{1}, \ldots, \sqrt{\lambda_{(n+p)(n+p)}} e_{n+p}\right] . } \\
& D_{2}=\left[\sqrt{\mu_{11}} e_{1}, \ldots, \sqrt{\mu_{1 p}} e_{1}, \sqrt{\mu_{21}} e_{2}, \ldots, \sqrt{\mu_{2 p}} e_{2}, \ldots, \sqrt{\mu_{(n+p) 1}} e_{(n+p)}, \ldots, \sqrt{\mu_{(n+p) p}} e_{n+p}\right], \\
& F_{2}=\operatorname{diag}\left(\varepsilon_{11}, \ldots, \varepsilon_{1 p}, \varepsilon_{21}, \ldots, \varepsilon_{2 p}, \varepsilon_{(n+p) 1}, \ldots, \varepsilon_{(n+p) p}\right),\left|\varepsilon_{i j}\right| \leq 1,1 \leq i \leq n+p, 1 \leq j \leq p, \\
& E_{2}^{T}=\left[\sqrt{\mu_{11}} \theta_{1}, \ldots, \sqrt{\mu_{1 p}} \theta_{p}, \sqrt{\mu_{21}} \theta_{1}, \ldots, \sqrt{\mu_{2 p}} \theta_{p}, \ldots, \sqrt{\mu_{(n+p) 1}} \theta_{1}, \ldots, \sqrt{\mu_{(n+p) p}} \theta_{p}\right] .
\end{aligned}
$$

Where, $e_{i}$ is the $i$ column vector of the order $n+p$ unit matrix $I, \theta_{i}$ is the $i$ column vector of the order $p$ unit matrix $I$, and $F_{1}^{T} F_{1} \leq I, F_{2}{ }^{T} F_{2} \leq I$. Therefore, the matrix $A\left(h_{k}, \tau_{k}\right)$ is described as $A=A_{0}+D_{1} F_{1} E_{1}$, the matrix $B\left(h_{k}, \tau_{k}\right)$ is described as $B=B_{0}+D_{2} F_{2} E_{2}$.

Therefore, the state model of the system can be finally transformed into

$$
z(k+1)=\left(A_{0}+D_{1} F_{1} E_{1}\right) z(k)+\left(B_{0}+D_{2} F_{2} E_{2}\right) u(k)
$$

Schur complement. Assume that $S_{11}$ is square matrix of $R \times R$, for symmetric matrix $S=\left[\begin{array}{cc}S_{11} & S_{12} \\ * & S_{22}\end{array}\right]$, the following conditions are equivalent:

(1) $S<0$

(2) $S_{22}<0, S_{11}-S_{12} S_{22}^{-1} S_{12}^{T}<0$

(3) $S_{11}<0, S_{22}-S_{12}^{T} S_{11}^{-1} S_{12}<0$

Lemma 1. Given the constant matrices $\Omega_{1}, \Omega_{2}, \Omega_{3}$, of appropriate dimensions, where $\Omega_{1}=\Omega_{1}^{T}$ and $\Omega_{2}=\Omega_{2}^{T}>0$, then

$$
\Omega_{1}+\Omega_{3}^{T} \Omega_{2}^{-1} \Omega_{3}<0
$$

if and only if

$$
\left[\begin{array}{cc}
\Omega_{1} & \Omega_{3}^{T} \\
\Omega_{3} & -\Omega_{2}
\end{array}\right]<0 \text { or }\left[\begin{array}{cc}
-\Omega_{2} & \Omega_{3} \\
\Omega_{3}^{T} & \Omega_{1}
\end{array}\right]<0 .
$$

Lemma 2 [17]. Given matrices $Y, H, E$ of appropriate dimensions and with $Y$ symmetric, then for all $F$ satisfying $F^{T} F \leq I$ and

$$
Y+H F E+E^{T} F^{T} H^{T}<0
$$

if and only if there exists $\varepsilon>0$ such that

$$
Y+\varepsilon H H^{T}+\varepsilon^{-1} E^{T} E<0 .
$$

\section{Main Results}

For system (11), define performance indicators:

$$
J=\sum_{k=2}^{\infty}\left(z^{T}(k) Q z(k)+u^{T}(k) R u(k)\right)
$$

where $Q$ and $R$ are positive definite matrices.

Next, we want to design a reliable guaranteed cost feedback control law

$$
u(k)=(K+\Delta K) z(k)
$$

such that for any acceptable range of uncertainties, the system

$$
z(k+1)=\left(A_{0}+D_{1} F_{1} E_{1}\right) z(k)+\left(B_{0}+D_{2} F_{2} E_{2}\right) u(k)
$$

can remain stable, and the cost function (12) satisfies $J \leq J^{*}$, where $J^{*}$ is a known constant.

In equation (13), $K$ is the gain of the controller, $\Delta K$ is the disturbance of the gain, and $\Delta K$ can be expressed as $\Delta K=D_{0} F_{0} E_{0}$, where $D_{0}$ and $E_{0}$ are the given appropriate dimension matrices, and $F_{0}$ is the unknown parameter matrix satisfying $F_{0}^{T} F \leq I$.

Definition 1 [18]. For the closed-loop system (11) and the performance index function (12), if there are gain matrices $K$ and $\Delta K$, so that the system (11) with any uncertain factors is asymptotically stable and satisfies $J \leq J^{*}$, then $J^{*}$ is the upper bound of the guaranteed performance index, and $u(k)=(K+\Delta K) z(k)$ is the guaranteed cost control law of the system (11) and the cost function (12).

Theorem 1. For the system (11), if there exist gain matrices $K, \Delta K$, positive-definite matrix $P$, satisfies the following inequalities:

$$
\begin{aligned}
& {[A+B(K+\Delta K)]^{T} P[A+B(K+\Delta K)]-P+Q} \\
& +(K+\Delta K)^{T} R(K+\Delta K)<0
\end{aligned}
$$

Then, the cost function (12) satisfies the following bound $J \leq J^{*}=z_{0}^{T} P z_{0}$.

Proof. Construct the Lyapunov function $V(k)=z_{k}^{T} P z_{k}$, and make the difference along any trajectory of the system:

$$
\begin{aligned}
\Delta V(k) & =V(k+1)-V(k)=z_{k+1}^{T} P z_{k+1}-z_{k}^{T} P z_{k} \\
& =z_{k}^{T}[A+B(K+\Delta K)]^{T} P[A+B(K+\Delta K)] z_{k}-z_{k}^{T} P z_{k}
\end{aligned}
$$


Since (15) holds, then

$$
\Delta V_{k}+z_{k}^{T}\left[Q+(K+\Delta K)^{T} R(K+\Delta K)\right] z_{k}<0,
$$

it is further deduced that

$$
z_{k}^{T}\left[Q+(K+\Delta K)^{T} R(K+\Delta K)\right] z_{k}=z_{k}^{T} Q z_{k}+u_{k}^{T} R u_{k}<-\Delta V_{k},
$$

So we know that $\Delta V(k)<0$.

Then, we sum the left and right ends of the above formula from $k=0$ to $k=\infty$, and we can get

$$
J=\sum_{k=0}^{\infty}\left[z_{k}^{T} Q z_{k}+u_{k}^{T} R u_{k}\right] \leq z_{0}^{T} P z_{0}
$$

It can be seen from this that the performance index is related to $z_{0}$. If $z_{0}$ is a random variable and satisfies $E\left\{z_{0} z_{0}{ }^{T}\right\}=I$, then the expected value of the system guaranteed performance index is

$$
E\left\{J^{*}\right\}=E\left\{z_{0}^{T} P z_{0}\right\}=\operatorname{tr} P
$$

Then, we will design the guaranteed performance controller that meets the expectation.

Theorem 2. For system (11) and performance metrics (12), if there are positive-definite matrices $X, Y$, and scalars $\varepsilon_{1}, \varepsilon_{2}, \varepsilon_{3}, \varepsilon_{4}, \varepsilon_{5}$, such that the following LMI is feasible:

$$
\left[\begin{array}{ccccccccc}
\psi 1 & A_{0} X+B_{0} Y & 0 & 0 & 0 & 0 & 0 & 0 & 0 \\
* & \psi 2 & X E_{1}^{T} & Y E_{2} & X & Y^{T} & X E_{0} & 0 & 0 \\
* & * & -\varepsilon_{2} I & 0 & 0 & 0 & 0 & 0 & 0 \\
* & * & * & -\varepsilon_{2} I & 0 & 0 & 0 & D_{0} E_{2}{ }^{T} & 0 \\
* & * & * & * & -Q^{-1} & 0 & 0 & 0 & 0 \\
* & * & * & * & * & -R^{-1} & 0 & 0 & E_{0}{ }^{T} \\
* & * & * & * & * & * & -\varepsilon_{3} I & 0 & 0 \\
* & * & * & * & * & * & * & -\varepsilon_{4} I & 0 \\
* & * & * & * & * & * & * & * & -\varepsilon_{5} I
\end{array}\right]<0
$$

Then $u(k)=Y X^{-1} z(k)$ is a control law of the system (11), whose performance index satisfies $J \leq J^{*}$, where

$$
\psi 1=-X+\varepsilon_{1} D_{1} D_{1}^{T}+\varepsilon_{1} D_{2} D_{2}^{T}+\varepsilon_{3} B_{0} D_{0} D_{0}{ }^{T} B_{0}^{T}, \psi 2=-X+\varepsilon_{4} E_{0} E_{0}{ }^{T}+\varepsilon_{5} D_{0} D_{0}^{T}
$$

Proof. Using the Schur complement, the (15) can be transformed into

$$
\left[\begin{array}{cc}
-P^{-1} & A+B(K+\Delta K) \\
* & -P+Q+(K+\Delta K)^{T} R(K+\Delta K)
\end{array}\right]<0
$$

Substituting $A=A_{0}+D_{1} F_{1} E_{1}$ and $B=B_{0}+D_{2} F_{2} E_{2}$ into (23), we can obtain

$$
\left[\begin{array}{cc}
-P^{-1} & A_{0}+D_{1} F_{1} E_{1}+\left(B_{0}+D_{2} F_{2} E_{2}\right)(K+\Delta K) \\
* & -P+Q+(K+\Delta K)^{T} R(K+\Delta K)
\end{array}\right]<0
$$

Splitting inequality (24), we get

$$
\begin{aligned}
& {\left[\begin{array}{cc}
-P^{-1} & A_{0}+B_{0}(K+\Delta K) \\
* & -P+Q+(K+\Delta K)^{T} R(K+\Delta K)
\end{array}\right]} \\
& +\left[\begin{array}{cc}
0 & E_{1} \\
0 & E_{2}(K+\Delta K)
\end{array}\right]^{T}\left[\begin{array}{cc}
F_{1} & 0 \\
0 & F_{2}
\end{array}\right]^{T}\left[\begin{array}{cc}
D_{1} & D_{2} \\
0 & 0
\end{array}\right]^{T} \\
& +\left[\begin{array}{cc}
D_{1} & D_{2} \\
0 & 0
\end{array}\right]\left[\begin{array}{cc}
F_{1} & 0 \\
0 & F_{2}
\end{array}\right]\left[\begin{array}{cc}
0 & E_{1} \\
0 & E_{2}(K+\Delta K)
\end{array}\right]<0
\end{aligned}
$$

Define 


$$
W=\left[\begin{array}{cc}
-P^{-1} & A_{0}+B_{0}(K+\Delta K) \\
* & -P+Q+(K+\Delta K)^{T} R(K+\Delta K)
\end{array}\right]
$$

According to Lemma 2, when there is a constant matrix $\operatorname{diag}\left\{\varepsilon_{1} I, \varepsilon_{2} I\right\}$, the inequality (25) can be equivalently transformed into

$$
\begin{aligned}
& W+\left[\begin{array}{cc}
\varepsilon_{1} I & 0 \\
0 & \varepsilon_{2} I
\end{array}\right]^{-1}\left[\begin{array}{cc}
0 & E_{1} \\
0 & E_{2}(K+\Delta K)
\end{array}\right]^{T}\left[\begin{array}{cc}
0 & E_{1} \\
0 & E_{2}(K+\Delta K)
\end{array}\right] \\
& +\left[\begin{array}{cc}
\varepsilon_{1} I & 0 \\
0 & \varepsilon_{2} I
\end{array}\right]\left[\begin{array}{cc}
D_{1} & D_{2} \\
0 & 0
\end{array}\right]\left[\begin{array}{cc}
D_{1} & D_{2} \\
0 & 0
\end{array}\right]^{T}<0
\end{aligned}
$$

By combining and sorting out (26), we can get:

$$
\left[\begin{array}{cc}
-P^{-1}+\varepsilon_{1}\left(D_{1} D_{1}{ }^{T}+D_{2} D_{2}{ }^{T}\right) & A_{0}+B_{0}(K+\Delta K) \\
* & -P+Q+(K+\Delta K)^{T} R(K+\Delta K)+\varepsilon_{2}{ }^{-1}\left(E_{1}{ }^{T} E_{1}+(K+\Delta K)^{T} E_{2}{ }^{T} E_{2}(K+\Delta K)\right.
\end{array}\right]<0
$$

For inequality (27), using Schur complement, we can get:

$$
\left[\begin{array}{cccccc}
-P^{-1}+\varepsilon_{1}\left(D_{1} D_{1}^{T}+D_{2} D_{2}{ }^{T}\right) & A_{0}+B_{0}(K+\Delta K) & 0 & 0 & 0 & 0 \\
* & -P & E_{1}^{T} & (K+\Delta K)^{T} E^{T} & I & (K+\Delta K)^{T} \\
* & * & -\varepsilon_{2} I & 0 & 0 & 0 \\
* & * & * & -\varepsilon_{2} I & 0 & 0 \\
* & * & * & * & -Q^{-1} & 0 \\
* & * & * & * & * & -R^{-1}
\end{array}\right]<0
$$

Letting $\Delta K=D_{0} F_{0} E_{0}$ in (28), we can obtain

$$
\left[\begin{array}{cccccc}
-P^{-1}+\varepsilon_{1}\left(D_{1} D_{1}{ }^{T}+D_{2} D_{2}{ }^{T}\right) & A_{0}+B_{0} K+A_{0} D_{0} F_{0} E_{0} & 0 & 0 & 0 & 0 \\
* & -P & E_{1}^{T} & K^{T} E_{2}{ }^{T}+\left(D_{0} F_{0} E_{0}\right)^{T} E_{2}{ }^{T} & I & K^{T}+D_{0} F_{0} E_{2} \\
* & * & -\varepsilon_{2} I & 0 & 0 & 0 \\
* & * & * & -\varepsilon_{2} I & 0 & 0 \\
* & * & * & * & -Q^{-1} & 0 \\
* & * & * & * & * & -R^{-1}
\end{array}\right]<0
$$

The above equation (29) is equivalent to

$$
\left.\left[\begin{array}{cccccc}
-P^{-1}+\varepsilon_{1}\left(D_{1} D_{1}{ }^{T}+D_{2} D_{2}{ }^{T}\right) & A_{0}+B_{0} K & 0 & 0 & 0 & 0 \\
* & -P & E_{1}^{T} & K^{T} E_{2}{ }^{T}+\left(D_{0} F_{0} E_{0}\right)^{T} E_{2}{ }^{T} & I & K^{T}+D_{0} F_{0} E_{2} \\
* & * & -\varepsilon_{2} I & 0 & 0 & 0 \\
* & * & * & -\varepsilon_{2} I & 0 & 0 \\
* & * & * & * & -Q^{-1} & 0 \\
* & * & * & * & * & -R^{-1}
\end{array}\right]+\left[\begin{array}{c}
A_{0} D_{0} \\
0 \\
0 \\
0 \\
0 \\
0
\end{array}\right]\left[\begin{array}{c}
0 \\
E_{0}^{T} \\
0 \\
F_{0}
\end{array}\right]^{T}\left[\left[\begin{array}{c}
A_{0} D_{0} \\
0 \\
0 \\
0 \\
0 \\
0 \\
0 \\
0
\end{array}\right]+\left[\begin{array}{c}
0 \\
E_{0}{ }^{T} \\
0 \\
0 \\
0 \\
0
\end{array}\right]\right]^{T}\right]^{T}
$$

Introducing scalar $\varepsilon_{3}$, using Lemma 2 and Schur complement, it can be obtained

$$
\left[\begin{array}{ccccccc}
\phi & A_{0}+B_{0} K & 0 & 0 & 0 & 0 & 0 \\
* & -P & E_{1}{ }^{T} & K^{T} E_{2}{ }^{T}+\left(D_{0} F_{0} E_{0}\right)^{T} E_{2}{ }^{T} & I & K^{T}+D_{0} F_{0} E_{2} & E_{0}{ }^{T} \\
* & * & -\varepsilon_{2} I & 0 & 0 & 0 & 0 \\
* & * & * & -\varepsilon_{2} I & 0 & 0 & 0 \\
* & * & * & * & -Q^{-1} & 0 & 0 \\
* & * & * & * & * & -R^{-1} & 0 \\
* & * & * & * & * & * & -\varepsilon_{3} I
\end{array}\right]<0
$$


where, $\phi=-P^{-1}+\varepsilon_{1}\left(D_{1} D_{1}{ }^{T}+D_{2} D_{2}{ }^{T}\right)+\varepsilon_{3} B_{0} D_{0} D_{0}{ }^{T} B_{0}{ }^{T}$.

Similarly, introducing scalars $\varepsilon_{4}$ and $\varepsilon_{5}$, by using Schur complement again, and by Lemma 2, we can get:

$$
\left[\begin{array}{ccccccccc}
\phi & A_{0}+B_{0} K & 0 & 0 & 0 & 0 & 0 & 0 & 0 \\
* & \varphi & E_{1}{ }^{T} & K^{T} E_{2}{ }^{T} & I & K^{T} & E_{0}{ }^{T} & 0 & 0 \\
* & * & -\varepsilon_{2} I & 0 & 0 & 0 & 0 & 0 & 0 \\
* & * & * & -\varepsilon_{2} I & 0 & 0 & 0 & D_{0}{ }^{T} E_{2}{ }^{T} & 0 \\
* & * & * & * & -Q^{-1} & 0 & 0 & 0 & 0 \\
* & * & * & * & * & -R^{-1} & 0 & 0 & E_{0}{ }^{T} \\
* & * & * & * & * & * & -\varepsilon_{3} I & 0 & 0 \\
* & * & * & * & * & * & * & -\varepsilon_{4} I & 0 \\
* & * & * & * & * & * & * & * & -\varepsilon_{5} I
\end{array}\right]<0
$$

where $\varphi=-P^{-1}+\varepsilon_{4} E_{0} E_{0}{ }^{T}+\varepsilon_{5} D_{0} D_{0}{ }^{T}$.

Multiply the left and right sides of the inequality (32) by $\Omega^{T}$ and $\Omega$, where

$$
\Omega=\operatorname{diag}\left\{I, P^{-1}, I, I, I, I, I, I, I\right\},
$$

and take $X=P^{-1}, Y=K X$, substitute them into the matrix inequality (32), after sorting out, the inequality (21) in theorem 2 is obtained, and the proof is completed. If there are feasible solutions to the inequality (21), it means that the system is still able to maintain asymptotic stability under the sampling period is uncertain, and a reliable guaranteed cost controller is $u_{k}=Y X^{-1} z_{k}$, and the corresponding performance index satisfies $J^{*}$.

Based on Theorem 2, the following optimization problems are given

$\min \operatorname{tr}(S)$

(i) (21),

(ii) $\left[\begin{array}{cc}X & I \\ I & S\end{array}\right]>0$,

Using Lemma 1, (ii) in (33) can be converted into $S-X^{-1}>0$. If there exist feasible solution $(X, S)$, the minimization of $\operatorname{Trace}(S)$ can guarantee the minimization of $\operatorname{Trace}(X)$, which ensures that a global optimum. The following will verify the feasibility through numerical simulation.

\section{Simulations}

Consider the following controlled system model

$$
\left\{\begin{array}{l}
\dot{x}(t)=\left[\begin{array}{lll}
0 & 1 & 0 \\
0 & 0 & 1 \\
0 & 0 & 0
\end{array}\right] x(t)+\left[\begin{array}{l}
0 \\
1 \\
0
\end{array}\right] u(t) \\
y(t)=\left[\begin{array}{lll}
1 & 1 & 0
\end{array}\right] x(t)
\end{array}\right.
$$

Assuming the bounded interval $h_{k} \in\left[\begin{array}{ll}0.1 & 0.5\end{array}\right]$, $\tau_{k} \in\left[\begin{array}{ll}0.05 & 0.1\end{array}\right]$. Using the method proposed in this chapter, the following interval matrix is obtained

$$
A_{0}=\left[\begin{array}{ccc}
1 & 0.3 & 0.0244 \\
0 & 1 & 0.075 \\
0 & 0 & 0
\end{array}\right], B_{0}=\left[\begin{array}{c}
0.507 \\
0.225 \\
1
\end{array}\right],
$$

taking $Q=\operatorname{diag}\{1,1,1\}, \mathrm{R}=0.01$.

The optimal solution can be obtained:

$$
\begin{gathered}
X=\left[\begin{array}{lll}
0.1871 & 0.0503 & 0.0266 \\
0.0503 & 0.2165 & 0.0303 \\
0.0266 & 0.0303 & 0.1812
\end{array}\right] \\
Y=\left[\begin{array}{lll}
0.0714 & -0.2007 & 0.1214
\end{array}\right] \\
S=\left[\begin{array}{ccc}
-20.0722 & -0.0229 & -0.0125 \\
-0.0229 & -20.0865 & -0.0143 \\
-0.0125 & -0.0143 & -20.0699
\end{array}\right] .
\end{gathered}
$$

The corresponding guaranteed performance controller is

$$
u_{k}=\left[\begin{array}{lll}
0.5856 & -1.1722 & 0.78
\end{array}\right] z_{k}
$$

and the upper bound of the corresponding closed-loop cost function is $J^{*}=23.08$.

These numerical results show that the matrix inequality presented in Theorem 2 has satisfactory solutions, and the state feedback control law can also be obtained. This indicates that the analysis and design method of the NCS with time-varying sampling period in this chapter is feasible, which can keep the system stable and satisfy certain performance indexes.

Let the initial value of the controlled system be $x(0)=[1,0.5,2]^{T}$, using MATLAB to simulate, the corresponding state response curve can be obtained. It can be seen from Figure 1 that the system is asymptotically stable. 


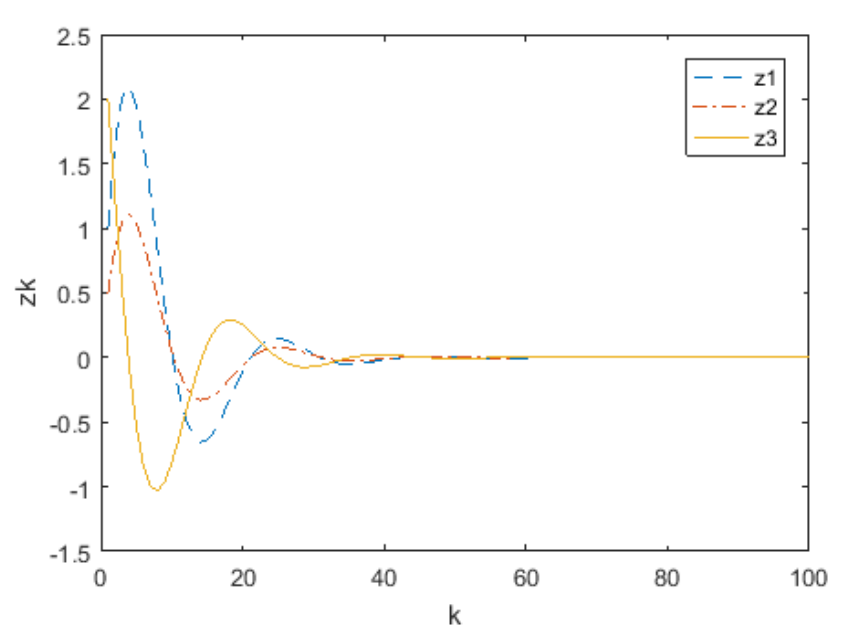

Figure 1. State response curve of closed-loop system.

\section{Conclusions}

In this paper, we have established a more general system model for the NCSs with time-varying sampling period. Based on LMI properties, the design method of guaranteed cost controller has been proposed, and the parametric representation of the controller has been given, so that the networked control system can keep normal operation when uncertainty factors fluctuate within the allowable range. In the design process, more free variables have been introduced to make the results less conservative. The feasibility solution has been given by using LMI toolbox, which has verified the effectiveness of the method. In the future research, we should consider and analyze the possible problems more comprehensively, such as packet loss, timing disorder, equipment failureand and so on. And the application of theoretical research in practical engineering, further verified in practice, will be the future research goal.

\section{References}

[1] Zhang D, Shi P, Wang Q G, et al. Analysis and synthesis of networked control systems: A survey of recent advances and challenges [J]. ISA Transactions, 2017, 66: 376-392.

[2] Wang Z W, Guo G. Research on basic problems of networked control systems [C]. Proceedings of the 33rd Chinese Control and Conference. Nanjing, China: IEEE, 2014: 5546-5550.

[3] Gupta R A, Chow M Y. Networked control system: overview and research trends [J]. IEEE Transactions on Industrial Electronics, 2010, 57 (7): 2527-2535.

[4] Park P G, Ko J W, Jeong C. Reciprocally convex approach to stability of systems with time-varying delays [J]. Automatica, 2011, 47 (1): 235-238.
[5] Tian Z D, Yi R, Wang G. A variable sampling period scheduling method for networked control system under resource constraints $[\mathrm{J}]$. Australian Journal of Electrical and Electronics Engineering, 2019, 16 (4): 289-304.

[6] Liu Y, Liu B. Modeling and switched control of networked control systems during time variable-period sampling $[\mathrm{J}]$. Application Research of Computers, 2018, 35 (01): 208-212.

[7] Mohajeri K, Madadi A, Tavassoli B. Tracking control with aperiodic sampling over networks with delay and dropout [J]. International Journal of Systems Science, 2021 (4): 1-16.

[8] Heemels W P M H, Teel A R, Wouw N V D, et al. Networked control systems with communication constraints: Tradeoffs between transmission intervals, delays and performance $[\mathrm{J}]$. IEEE Transaction Automatic Control, 2010, 55 (8): 1781-1796.

[9] Fridman E. A refined input delay approach to sampled-data control [J]. Automatica, 2010, 46 (2): 421- 427.

[10] Hua C, Zhang L, Guan X. Memoryless state feedback control for uncertain nonlinear time-delay system [M]. Springer Singapore, 2018.

[11] Zhang L, Jia M, Yang H, et al. Memory state feedback and robust fault-tolerant control for a class of time-delays switched fuzzy systems with nonlinear perturbation [C]// 2019 Chinese Control and Decision Conference (CCDC). IEEE, 2019.

[12] Lin W, Zhao C. Global stabilization by memoryless feedback for nonlinear systems with a small input delay and large state delays [J]. IEEE Transactions on Automatic Control, 2020, 99: $1-1$.

[13] Antonio S. Computer control under time-varying sampling period: An LMI gridding approach [J]. Automatica, 2005, 41 (12): 2077-2082.

[14] Wang Y L, Yang G H. Hळ control of networked control system with time-varying sampling period [J]. Information and Control, 2007 (03): 278-284.

[15] Qu Z F, Du Z B. Fuzzy sampled-data optimal control for nonlinear systems with time-varying dalay $[\mathrm{J}]$. Control and Decision, 2018, 33 (11): 2069-2072.

[16] Zhao Y, Wang Y L. Fault detection for nonlinear networked control systems with time varying sampling period and multi-packet transmission [J]. Computer Measurement and Control, 2016, 024 (005): 55-58, 66.

[17] Barmish B R. Necessary and sufficient conditions for quadratic stability of an uncertain system [J]. Journal of Optimization Theory and Applications, 1985, 46: 399-408.

[18] Yu L. Robust control: an LMI approach [M]. Beijing: Tsinghua University Press, 2002: 214-267. 